\title{
Comparison of clinical and microbiological diagnoses for older adults with COVID-19 in Wuhan: a retrospective study
}

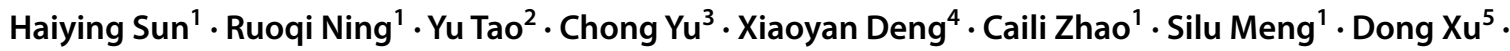 \\ Fangxu Tang ${ }^{1} \mathbb{D}$
}

Received: 20 May 2020 / Accepted: 29 June 2020 / Published online: 8 July 2020

(c) Springer Nature Switzerland AG 2020

\begin{abstract}
Background The potential differences between a clinical diagnosis of coronavirus disease 2019 (COVID-19) (i.e., symptoms without positive virus test) and a microbiological diagnosis (i.e., positive virus test results) of COVID-19 are not known. Aims This study explored the differences between the two types of COVID-19 diagnosis among older patients in terms of clinical characteristics and outcomes.

Methods A total of 244 inpatients aged $\geq 60$ years with COVID-19 were included in this study, of whom 52 were clinically diagnosed and 192 were microbiologically diagnosed. Clinical and laboratory data on hospital admission and outcomes (discharged or died in hospital) of all patients were retrieved from medical records retrospectively. Patients who met the criteria for clinical diagnosis with negative virus test results were assigned to the clinical diagnosis group, whereas those with positive virus test results were assigned to the microbiological diagnosis group. After univariate analyses, two propensity score analyses [i.e., covariate adjustment using propensity score (CAPS) and propensity score matching (PSM)] were conducted to control bias.

Results The clinical and microbiological diagnosis groups demonstrated significant differences in outcomes and in the majority of laboratory findings. After propensity score analyses, many differences between the two groups disappeared and the rate of mortality had no statistically significant difference ( $P=0.318$ and 0.828 for CAPS and PSM, respectively). Conclusions Patients with similar signs, symptoms, and laboratory and imaging findings as confirmed COVID-19 cases may have a similar mortality risk, regardless of the virus test results, and require timely intervention to reduce their mortality.
\end{abstract}

Keywords Clinical diagnosis $\cdot$ COVID-19 $\cdot$ Microbiological diagnosis $\cdot$ Older patients $\cdot$ SARS-CoV-2

\section{Introduction}

At the end of December 2019, cases of an acute pneumonia of unknown origin were detected in Wuhan, Hubei Province, China [1,2]. Following the unremitting efforts of clinicians and scientists, the pathogen was isolated and identified as

Haiying Sun and Ruoqi Ning contributed equally to this work.

Fangxu Tang

tfx19870521@sina.com

1 Department of Obstetrics and Gynecology, Tongji Hospital, Tongji Medical College, Huazhong University of Science and Technology, 1095 Jiefang Avenue, Wuhan 430030, Hubei, China

2 Department of Pulmonary and Critical Care Medicine, Tongji Hospital, Tongji Medical College, Huazhong University of Science and Technology, Wuhan, Hubei, China severe acute respiratory syndrome coronavirus 2 (SARSCoV-2) [3]. The disease caused by it was officially named as coronavirus disease 2019 (COVID-19) by the World Health Organization (WHO) [2]. Because of the global outbreak and rapidly increasing numbers of patients and deaths, the WHO has declared COVID-19 as a worldwide pandemic and a public health emergency of international concern.

3 Department of Nephrology, Tongji Hospital, Tongji Medical College, Huazhong University of Science and Technology, Wuhan, Hubei, China

4 Department of Cardiovascular Medicine, Tongji Hospital, Tongji Medical College, Huazhong University of Science and Technology, Wuhan, Hubei, China

5 Department of Infectious Diseases, Tongji Hospital, Tongji Medical College, Huazhong University of Science and Technology, Wuhan, Hubei, China 
Recognizing and diagnosing individuals with COVID-19 quickly and providing them with treatment and quarantine in time are becoming a huge challenge worldwide.

As COVID-19 is a respiratory infectious disease caused by an RNA virus, real-time reverse transcriptase polymerase chain reaction (RT-PCR) is regarded as the gold standard method for the diagnosis of COVID-19. However, because of some as yet unknown factors, the detection rate for SARSCoV-2 using RT-PCR is not high [4, 5]. There have also been several recent reports about false-negative results of nucleic acid tests for COVID-19 [6,7]. To provide patients with timely treatment and to block viral transmission as soon as possible, the National Health Commission of the People's Republic of China has revised the diagnostic criteria in the fifth version of the guideline for diagnosis and treatment of COVID-19 [8]. A new type of diagnosis, namely clinical diagnosis, was performed in Hubei Province, which is the area with the highest prevalence of COVID-19 in China. Clinical diagnosis of COVID-19 is based mainly on clinical, epidemic, and radiologic features, regardless of the RT-PCR results, whereas microbiological diagnosis is based mainly on SARS-CoV-2 test results. It is still unknown whether there are differences between clinically and microbiologically (i.e. positive RT-PCR results for SARS-CoV-2 RNA) diagnosed patients.

Recent studies have demonstrated that older age was a risk factor for death from COVID-19 [9, 10]. Because of the progressive aging of the population, the diagnosis and treatment for older adults with COVID-19 should be brought to the forefront worldwide. Therefore, this study aimed at exploring the differences between the two types of diagnosis among older patients in terms of clinical characteristics on admission and outcomes (i.e., discharged or died in the hospital) and at contributing toward optimizing the diagnosis and treatment of these patients.

\section{Methods}

\section{Definitions}

Clinical diagnosis Patients from epidemic areas who showed clinical (i.e., fever or respiratory symptoms) and laboratory (i.e., normal or decreased white blood cell counts, or decreased lymphocyte counts) features as well as imaging findings of viral pneumonia (i.e., ground-glass opacities, multifocal patchy consolidation, infiltration shadowing and interstitial abnormalities with a peripheral distribution) were clinically diagnosed as having COVID-19 by two experienced respiratory physicians [8].

Microbiological diagnosis: Patients who met the criteria for clinical diagnosis with positive evidence of SARS-CoV-2
RNA were microbiologically diagnosed as having COVID19 [8].

\section{Study design and participants}

Our study was based on retrospective observation of inpatients in the Sino-French New City Branch of Tongji Hospital, which was an assigned hospital for the treatment of severe and critical patients with COVID-19 in Wuhan. It has 23 wards (including 2 intensive care units) with 1085 beds. The study's observational end point was either death due to COVID-19 during hospitalization or eligible discharge [8] between January 29, 2020 and March 5, 2020. Patients aged 60 years or older with definite outcomes (i.e., discharged or died in hospital) by March 5, 2020 were included in our study. All patients provided nasopharyngeal or oropharyngeal swab samples upon admission, and the SARS-CoV-2 tests were repeated during the course of the hospitalization. The presence of SARS-CoV-2 in the patients' specimens was detected by RT-PCR using kits produced by DAAN GENE Co., Ltd of Sun Yat-Sen University. Participants who met the criteria for clinical diagnosis with only negative virus test results (including the tests at admission and those repeated during hospitalization) were allocated to the clinical diagnosis group, and those with positive virus test results were allocated to microbiological diagnosis groups.

\section{Data collection}

Data on each patient's clinical characteristics, laboratory and imaging findings on admission, and outcomes were collected and reviewed by at least two experienced clinicians through assessment of electronic medical records. A trained team of researchers analyzed the data, including symptoms, signs, laboratory test results, and outcomes. Peripheral oxygen saturation $\left(\mathrm{SpO}_{2}\right)$ was measured in the patient's oxygenabsorbing state.

\section{Statistics}

Comparison of categorical variables (except outcomes) between the two groups was evaluated using the Pearson Chi-square test with continuity correction or Fisher' s exact test. The Mann-Whitney $U$ test was used for continuous variables. Propensity score analyses, including covariate adjustment using propensity score (CAPS) and propensity score matching (PSM), were used to control the bias of confounding factors. Age, white blood cell (WBC) count, and lymphocyte (LYM) count were included in the propensity score model (using a logistic regression), which were the most important risk factors for death in the hospital among older patients with COVID-19 [11]. WBC count and LYM count were also the most important laboratory indicators of 
clinical diagnosis criteria for COVID-19. Continuous variables were treated as continuous measures in our analysis. Samples with missing values in the three variables above were excluded from the propensity score model. Univariate logistic regression was used to estimate the differences in outcomes between the two groups before propensity score analyses and after PSM. The PSM was performed at a ratio of $1: 1$ with 0.01 caliper and random sampling without replacement. Only matched samples were included in the PSM analysis. Statistical analyses were performed using SPSS 21.0. Statistical tests were considered significant when two-sided $P$ values were less than 0.05 .

\section{Results}

A total of 244 older patients were included in this study, with 52 patients undergoing clinical diagnosis and 192 patients, microbiological diagnosis. Of the patients, 16 (30.8\%) and 107 (55.7\%) in the clinical and microbiological diagnosis groups, respectively, were discharged. The median patient age was 68 years for the clinical diagnosis group and 70 years for microbiological diagnosis group $(P=0.286$; Table 1$)$. Male individuals accounted for $69.2 \%$ of patients in the clinical diagnosis group and $50.5 \%$ in the microbiological group, a between-group difference that was statistically significant $(P=0.016)$. The majority of patients in both the clinical and microbiological diagnosis groups had fever $(86.5 \%, 86.5 \%, P=0.988)$ and respiratory symptoms $(86.5 \%, 89.1 \%, P=0.612)$, especially cough $(69.2 \%$, $74.5 \%, P=0.448)$. The proportion of patients with gastrointestinal symptoms in the clinical diagnosis group was higher than that in the microbiological group ( $48.1 \%$ vs. $29.2 \%$, $P=0.010$ ). The between-group distribution of the WBC count was statistically significantly different (median value 10.17 vs. $6.07, P<0.001)$. The $P$ value of the distribution difference for the LYM count was 0.053 . The erythrocyte sedimentation rate (ESR) distribution was similar between the two groups $(P=0.864)$. Other laboratory findings, such as amino-terminal pro-brain natriuretic peptide (NTproBNP), procalcitonin, high-sensitivity cardiac troponin I (hsTnI), D-dimer, alanine aminotransferase (ALT), aspartate aminotransferase (AST), creatinine, eGFR, high-sensitivity C reactive protein (hsCRP), serum ferritin, and interleukin-6 (IL-6) levels, were significantly different $(P<0.05)$ between the groups.

Propensity score analyses were applied to balance the confounding factors between the two groups. Older age, higher WBC count, and lower LYM count were previously identified as three important prognostic factors for COVID19 [11]. The propensity score was calculated using a logistic regression model based on these three factors. Both the clinical and microbiological diagnosis groups contained one case each with missing values for WBC count and LYM count, and those cases were excluded from the propensity score model. A total of 92 patients were included in the PSM analysis, with 46 patients from each group (Table 2). The median ages were 69 and 70 years for the matched clinical and microbiological groups, respectively, and the majority of patients in the two matched groups were male $(69.5 \%$ vs. $56.5 \%, P=0.195)$. After matching, there were no statistical differences in most variables between the two groups, especially the laboratory variables $(P>0.05)$. The $P$ value of D-dimer was 0.047 [median (IQR) 3.60 (1.22-21.00) vs. 1.34 (0.74-15.13)]. Among the comorbidities, hypertension was still the most common, with the proportion of patients with hypertension at 63\% (29/46) and 50\% (23/46) in the matched clinical and microbiological diagnosis groups $(P=0.207)$. However, gastrointestinal symptoms were significantly different between the matched clinical and microbiological groups $(P=0.001)$. The proportion of patients with gastrointestinal symptoms were $47.8 \%$ and $15.2 \%$, respectively. Diarrhea was a common symptom, and the distribution of this symptom was also significantly different between the two matched groups $(41.3 \%$ vs. $13.0 \%$, $P=0.002$ ).

The disease outcomes between the two groups were significantly different $(P=0.002)$ before propensity score analyses (Table 3). However, after controlling for bias by CAPS, the difference in outcomes between the two groups disappeared $(P=0.318)$ (Table 3$)$. In PSM samples, 16 patients were discharged, and 30 patients died in the matched clinical diagnosis group, while 17 were discharged and 29 died in the matched microbiological diagnosis group. The outcomes between the two groups after PSM were similar $[P=0.828$, OR $1.099,95 \%$ CI $(0.469,2.578)]$ (Table 3$)$.

\section{Discussion}

In this retrospective study, we found that there were many differences in clinical features, laboratory findings, and patient outcomes between the two types of COVID-19 diagnoses. Mortality in the clinical diagnosis group was significantly higher than that in the microbiological diagnosis group. Treatments for COVID-19 were based on patients' conditions according to the guidelines and were not related to the type of diagnosis. This revealed that patients with a clinical diagnosis of COVID-19 had more severe disease than those with a microbiological diagnosis. However, after PSM, most of the differences disappeared. Of note, the mortalities of the two groups were no longer significantly different after CAPS or PSM analysis. Gastrointestinal symptoms and consciousness disorders were significantly different before and after matching in the two clinical and microbiological groups. 
Table 1 Differences of characteristics between clinically and microbiologically diagnosed cases (total samples) before propensity score analyses

\begin{tabular}{|c|c|c|c|}
\hline Characteristics & Clinical $^{\mathrm{a}}$ & Microbiological $^{\mathrm{a}}$ & $P$ value \\
\hline \multicolumn{4}{|l|}{ Demographics } \\
\hline Age (years) & $68(64-75)$ & $70(65-76)$ & 0.286 \\
\hline Gender & & & 0.016 \\
\hline Male & $36(69.2)$ & $97(50.5)$ & \\
\hline Female & $16(30.8)$ & $95(49.5)$ & \\
\hline \multicolumn{4}{|l|}{ Vital signs } \\
\hline $\mathrm{SpO}_{2}(\%)$ & $95(85-98)$ & $96(88-98)$ & 0.711 \\
\hline Pulse rate (bpm) & $89(80-102)$ & $89(81-102)$ & 0.858 \\
\hline Respiratory rate (bpm) & $23(20-30)$ & $20(20-24)$ & 0.025 \\
\hline Consciousness disorders & $16(30.8)$ & $16(8.3)$ & $<0.001$ \\
\hline \multicolumn{4}{|l|}{ Symptoms } \\
\hline Fever & $45(86.5)$ & $166(86.5)$ & 0.988 \\
\hline Temperature-highest $\left({ }^{\circ} \mathrm{C}\right)$ & $38.5(38.0-39.0)$ & $38.4(37.8-39.0)$ & 0.261 \\
\hline Respiratory symptoms & $45(86.5)$ & $171(89.1)$ & 0.612 \\
\hline Cough & $36(69.2)$ & $143(74.5)$ & 0.448 \\
\hline Dyspnea & $35(67.3)$ & $122(63.5)$ & 0.615 \\
\hline Gastrointestinal symptoms & $25(48.1)$ & $56(29.2)$ & 0.010 \\
\hline Diarrhea & $21(40.4)$ & $51(26.6)$ & 0.053 \\
\hline Abdominal pain & $4(7.7)$ & $6(3.1)$ & 0.227 \\
\hline \multicolumn{4}{|l|}{ Histories } \\
\hline Hypertension & $32(61.5)$ & $106(55.5)$ & 0.436 \\
\hline Diabetes & $8(15.4)$ & $43(22.5)$ & 0.263 \\
\hline Coronary heart disease & $10(19.2)$ & $25(13.1)$ & 0.263 \\
\hline Respiratory diseases & $4(7.7)$ & $20(10.5)$ & 0.552 \\
\hline \multicolumn{4}{|l|}{ Laboratory findings } \\
\hline WBC count $\left(\times 10^{9} / \mathrm{L}\right)$ & $10.17(6.20-12.94)$ & $6.07(4.73-9.34)$ & $<0.001$ \\
\hline LYM count $\left(\times 10^{9} / \mathrm{L}\right)$ & $0.60(0.44-0.96)$ & $0.73(0.51-1.12)$ & 0.053 \\
\hline NT-proBNP $\left(\times 10^{2} \mathrm{pg} / \mathrm{mL}\right)$ & $8.00(1.75-36.14)$ & $3.22(1.49-8.24)$ & 0.008 \\
\hline Procalcitonin (ng/mL) & $0.31(0.10-1.31)$ & $0.10(0.04-0.32)$ & $<0.001$ \\
\hline hsTnI (pg/mL) & $29.3(7.4-425.0)$ & $11.4(4.7-30.5)$ & 0.001 \\
\hline D-dimer $(\mu \mathrm{g} / \mathrm{mL}$ FEU) & $4.47(1.26-21.00)$ & $1.34(0.59-3.26)$ & $<0.001$ \\
\hline ALT (U/L) & $28.0(19.0-46.0)$ & $23.0(15.0-38.0)$ & 0.047 \\
\hline AST (U/L) & $45.0(29.0-65.0)$ & $33.0(24.0-52.0)$ & 0.008 \\
\hline Creatinine $(\mu \mathrm{mol} / \mathrm{L})$ & $87.0(72.0-118.0)$ & $76.0(57.0-94.0)$ & 0.009 \\
\hline eGFR $\left(\mathrm{mL} / \mathrm{min} / 1.73 \mathrm{~m}^{2}\right)$ & $71.8(48.9-89.0)$ & $80.3(60.9-92.5)$ & 0.037 \\
\hline hsCRP (mg/L) & $103.2(47.5-169.3)$ & $65.0(27.4-114.1)$ & 0.001 \\
\hline $\operatorname{ESR}(\mathrm{mm} / \mathrm{H})$ & $36.0(20.0-63.0)$ & $37.0(20.0-59.0)$ & 0.864 \\
\hline Serum ferritin $\left(\times 10^{2} \mu \mathrm{g} / \mathrm{L}\right)$ & $13.50(6.88-24.10)$ & $7.73(4.56-16.46)$ & 0.010 \\
\hline Interleukin-6 (pg/mL) & $58.01(31.07-131.95)$ & $30.37(5.61-77.65)$ & 0.005 \\
\hline
\end{tabular}

WBC white blood cells, $L Y M$ lymphocyte, NT-proBNP amino-terminal pro-brain natriuretic peptide, $h s T n I$ high-sensitivity cardiac troponin I, $A L T$ alanine aminotransferase, $A S T$ aspartate aminotransferase, $e G F R$ estimated glomerular filtration rate, $h S C R P$ high-sensitivity C-reactive protein, $E S R$ erythrocyte sedimentation rate

${ }^{\text {a }}$ Data presented as median (IQR) and $n(\%)$ for continuous and categorical variables, respectively, unless otherwise indicated
A false-negative result of SARS-CoV-2 detection may delay the diagnosis of COVID-19. Since the outbreak of COVID-19, detection of etiology has played a decisive role in the diagnosis of infected people. Scientists from China have obtained and uploaded the full-length genomic sequence of SARS-CoV-2 [12], and several nucleic acid detection kits based on this sequence have been developed. However, differences in sensitivity and specificity have been found on clinical use of different testing kits. For instance, the incidence of false-negative virus test results was high 
Table 2 Characteristics of clinically and microbiologically diagnosed cases after propensity score matching

\begin{tabular}{|c|c|c|c|}
\hline Characteristics & Clinical $^{\mathrm{a}}$ & Microbiological $^{\mathrm{a}}$ & $P$ value \\
\hline \multicolumn{4}{|l|}{ Demographics } \\
\hline Age (years) & $69(64-78)$ & $70(64-76)$ & 0.944 \\
\hline \multicolumn{4}{|l|}{ Gender } \\
\hline Male & $32(69.6)$ & $26(56.5)$ & 0.195 \\
\hline Female & $14(30.4)$ & $20(43.5)$ & \\
\hline \multicolumn{4}{|l|}{ Vital signs } \\
\hline $\mathrm{SpO}_{2}(\%)$ & $95(85-97)$ & $94(82-97)$ & 0.484 \\
\hline Pulse rate (bpm) & $89(79-101)$ & $90(81-111)$ & 0.761 \\
\hline Respiratory rate (bpm) & $22(20-30)$ & $21(20-28)$ & 0.547 \\
\hline Consciousness disorders & $13(28.3)$ & $4(8.7)$ & 0.016 \\
\hline \multicolumn{4}{|l|}{ Symptoms } \\
\hline Fever & $40(87.0)$ & $38(82.6)$ & 0.562 \\
\hline Temperature-highest $\left({ }^{\circ} \mathrm{C}\right)$ & $38.5(38.0-39.0)$ & $38.5(37.8-38.9)$ & \\
\hline Respiratory symptoms & $40(87.0)$ & $40(87.0)$ & 1.000 \\
\hline Cough & $33(71.7)$ & $28(60.9)$ & 0.270 \\
\hline Dyspnea & $30(65.2)$ & $31(67.4)$ & 0.825 \\
\hline Gastrointestinal symptoms & $22(47.8)$ & $7(15.2)$ & 0.001 \\
\hline Diarrhea & $19(41.3)$ & $6(13.0)$ & 0.002 \\
\hline Abdominal pain & $3(6.5)$ & $0(0.0)$ & 0.242 \\
\hline \multicolumn{4}{|l|}{ Histories } \\
\hline Hypertension & $29(63.0)$ & $23(50.0)$ & 0.207 \\
\hline Diabetes & $7(15.2)$ & $9(19.6)$ & 0.582 \\
\hline Coronary heart disease & $8(17.4)$ & $8(17.4)$ & 1.000 \\
\hline Respiratory diseases & $4(8.7)$ & $8(17.4)$ & 0.216 \\
\hline \multicolumn{4}{|l|}{ Laboratory findings } \\
\hline WBC count $\left(\times 10^{9} / \mathrm{L}\right)$ & $9.13(6.10-12.26)$ & $9.33(5.89-11.72)$ & 0.941 \\
\hline LYM count $\left(\times 10^{9} / \mathrm{L}\right)$ & $0.64(0.43-0.98)$ & $0.66(0.34-1.10)$ & 0.947 \\
\hline NT-proBNP $\left(\times 10^{2} \mathrm{pg} / \mathrm{mL}\right)$ & $7.90(1.72-35.76)$ & $6.01(2.03-13.75)$ & 0.799 \\
\hline Procalcitonin (ng/mL) & $0.28(0.09-1.18)$ & $0.18(0.07-0.68)$ & 0.217 \\
\hline hsTnI (pg/mL) & $35.2(8.1-425.0)$ & $21.0(6.6-63.7)$ & 0.199 \\
\hline D-dimer $(\mu \mathrm{g} / \mathrm{mL}$ FEU $)$ & $3.60(1.22-21.00)$ & $1.34(0.74-15.13)$ & 0.047 \\
\hline ALT (U/L) & $28.0(19.0-41.0)$ & $28.0(17.0-41.0)$ & 0.623 \\
\hline AST (U/L) & $44.0(29.0-58.0)$ & $36.5(26.0-56.0)$ & 0.269 \\
\hline Creatinine $(\mu \mathrm{mol} / \mathrm{L})$ & $86.5(72.0-117.0)$ & $87.0(65.0-103.0)$ & 0.555 \\
\hline eGFR $\left(\mathrm{mL} / \mathrm{min} / 1.73 \mathrm{~m}^{2}\right)$ & $71.8(50.0-85.0)$ & $77.6(51.3-91.2)$ & 0.458 \\
\hline hsCRP (mg/L) & $103.3(51.1-169.3)$ & $71.0(36.7-12.6)$ & 0.034 \\
\hline $\operatorname{ESR}(\mathrm{mm} / \mathrm{H})$ & $36.0(20.0-63.0)$ & $41.0(18.0-60.0)$ & 0.983 \\
\hline Serum ferritin $\left(\times 10^{2} \mu \mathrm{g} / \mathrm{L}\right)$ & $12.38(6.64-22.47)$ & $10.72(5.14-19.44)$ & 0.379 \\
\hline Interleukin-6 (pg/mL) & $55.07(26.99-103.00)$ & $31.99(9.99-113.40)$ & 0.168 \\
\hline
\end{tabular}

WBC white blood cells, $L Y M$ lymphocyte, NT-proBNP amino-terminal pro-brain natriuretic peptide, $h s T n I$ high-sensitivity cardiac troponin I, ALT alanine aminotransferase, AST aspartate aminotransferase, eGFR estimated glomerular filtration rate, $h S C R P$ high-sensitivity C-reactive protein, $E S R$ erythrocyte sedimentation rate

${ }^{\text {a }}$ Data presented as median (IQR) and $n(\%)$ for continuous and categorical variables, respectively, unless otherwise indicated
[13]. As a result, some patients might be misdiagnosed when the positive result of virus test is a necessary criterion for diagnosis of COVID-19. Several explanations could account for the high false-negative rate. Currently, nasal and pharyngeal swabs are the main methods to obtain samples for nucleic acid testing for SARS-CoV-2. However, a recent study revealed that nasal and pharyngeal swabs demonstrated a poor positive rate, while the bronchoalveolar lavage fluid exhibited a high detection rate [14]. As COVID-19 is a virus that can infect the upper and lower respiratory 
Table 3 The outcomes of clinically and microbiologically diagnosed cases before and after propensity score analyses

\begin{tabular}{|c|c|c|c|c|c|c|}
\hline \multirow[t]{2}{*}{ Grouping } & \multicolumn{3}{|c|}{ Outcomes } & \multirow[t]{2}{*}{$P$ value } & \multirow[t]{2}{*}{ OR } & \multirow[t]{2}{*}{$95 \% \mathrm{CI}$} \\
\hline & Total & Discharged & Dead & & & \\
\hline $\begin{array}{l}\text { Before propensity } \\
\text { score analysis }\end{array}$ & & & & 0.002 & 2.832 & $1.473-5.448$ \\
\hline Clinical & 52 & 16 & 36 & & & \\
\hline Microbiological & 192 & 107 & 85 & & & \\
\hline CAPS & & & & 0.318 & 1.490 & $0.681-3.262$ \\
\hline Clinical & $51^{\mathrm{a}}$ & 16 & $35^{\mathrm{a}}$ & & & \\
\hline Microbiological & $191^{\mathrm{b}}$ & 107 & $84^{\mathrm{b}}$ & & & \\
\hline PSM & & & & 0.828 & 1.099 & $0.469-2.578$ \\
\hline Clinical & 46 & 16 & 30 & & & \\
\hline Microbiological & 46 & 17 & 29 & & & \\
\hline
\end{tabular}

$O R$ odds ratio, $C I$ confidence interval of $\mathrm{OR}, C A P S$ covariate adjustment using propensity score, $P S M$ propensity score matching

${ }^{a}$ One case of clinical diagnosis group was excluded from CAPS analysis for containing missing data in WBC count and LYM count

${ }^{\mathrm{b}}$ One case of microbiological diagnosis group was excluded from CAPS analysis for containing missing data in WBC count and LYM count tract simultaneously, there may be differences in viral load between samples from different parts of the respiratory tract, which could in turn cause a disparity in the detection rate. The collection of nasal and pharyngeal swabs may cause patients to be slightly uncomfortable. Furthermore, collection of such samples usually requires a certain amount of cooperation from patients. It is difficult to collect nasal and pharyngeal swabs from some patients, such as those who face difficulties in communication, like older patients (e.g., the participants in our study), which will influence the detection rate of the virus. In addition, as mentioned above, testing kit performance is also associated with the microbiological test outcome.

There was a certain proportion of patients who did not have respiratory symptoms but had gastrointestinal symptoms [10, 15]. In our study, the presence of gastrointestinal symptoms was statistically different between the clinical and microbiological groups before and after PSM, and the proportion of patients with gastrointestinal symptoms was greater in the clinical diagnosis group. Our previous study results showed that gastrointestinal symptoms were not a risk factor for death [11], but some studies observed that gastrointestinal symptoms were related to worse outcomes [16]. Gastrointestinal symptoms may mislead or delay the diagnosis of COVID-19, especially in patients mainly presenting with gastrointestinal symptoms [17]. The positivity rate for virus tests of feces samples in patients with gastrointestinal symptoms was much higher than the average [13]. The viral load of the respiratory tract may not be high enough to be detectable in these patients, which may also explain the negative results of their nasopharyngeal swabs. This suggests that for patients with nasopharyngeal swab-negative results, attention should be paid to their gastrointestinal symptoms, and anal swab virus detection should be considered, when possible.

Before propensity score analyses, the clinical and laboratory features of patients in the clinical diagnosis group were more severe than those of patients in the microbiological group on admission. In particular, consciousness disorders, and increased WBC count, procalcitonin and D-dimer levels are associated with worse outcomes [11]. After 1:1 propensity score matching based on age, WBC, and LYM count, which were identified as important prognostic factors in our previous work [11] and also reported by other recent studies $[9,15]$, many differences in the clinical features and laboratory findings disappeared. WBC and LYM counts were also the most important indicators in clinical diagnosis criteria in the 5 th version of the guideline for diagnosis and treatment of COVID-19. At the same time, the two matched groups demonstrated similar mortality. This means that patients with similar signs, symptoms, and laboratory findings may have similar outcomes, regardless of the results of RT-PCR for viral RNA detection. Therefore, the higher mortality in the clinical diagnosis group before PSM may mainly be because of the more severe disease state than that of patients in the microbiological group on admission.

Although the current study is limited by the small sample size after PSM, it is the first to investigate the differences in clinical and microbiological diagnoses of COVID-19. The negative result achieved from PSM was confirmed by CAPS, thus proving its authenticity.

For clinicians treating COVID-19 in epidemic areas where the supply of virus detection kits is insufficient or the performance of kits is poor, older patients with clinical 
features of COVID-19, especially gastrointestinal symptoms such as diarrhea, should be paid more attention when making medical decisions. Even if the virus tests are negative, older adults eligible for clinical diagnosis of COVID-19 may require timely intervention to reduce mortality.

Author contributions Concept and design: FT, and HS. Data collection: RN, YT, CY, XD, CZ, and SM. Analysis and interpretation of data: HS, RN, FT, DX. Manuscript writing: all authors. Final approval of manuscript: all authors.

Funding None.

Availability of data and materials All data generated or analyzed during this study are included in this published article.

\section{Compliance with ethical standards}

Conflict of interest The authors declare that they have no conflicts of interest.

Ethics approval This study followed the Helsinki Declaration of the World Medical Association. The study was approved by the Ethics Committee of Tongji Hospital of Tongji Medical College of Huazhong University of Science and Technology (TJ-IRB20200505).

Statement of human and animal rights The present study followed the ethical standards for Humans and animal rights.

Informed consent The requirement for written informed consent was waived by the Ethics Committee of Tongji Hospital of Tongji Medical College of Huazhong University of Science and Technology.

Consent for publication The publication was approved and the requirement for written informed consent was waived by the Ethics Committee of Tongji Hospital of Tongji Medical College of Huazhong University of Science and Technology.

Code availability Not applicable.

\section{References}

1. Chen ATC, Coura-Filho GB, Rehder MHH (2020) Clinical characteristics of Covid-19 in China. N Engl J Med 382:1860. https:// doi.org/10.1056/NEJMc2005203

2. Coronavirus disease (COVID-19) Pandemic (2020) World Health Organization. https://www.who.int/emergencies/diseases/novel -coronavirus-2019. Accessed 14 May 2020

3. Zhu N, Zhang D, Wang W et al (2020) A novel coronavirus from patients with pneumonia in China, 2019. N Engl J Med 382:727733. https://doi.org/10.1056/NEJMoa2001017

4. Corman VM, Landt O, Kaiser M et al (2020) Detection of 2019 novel coronavirus $(2019-\mathrm{nCoV})$ by real-time RT-PCR. Euro
Surveill. https://doi.org/10.2807/1560-7917.ES.2020.25.3.20000 45

5. Zhang N, Wang L, Deng X et al (2020) Recent advances in the detection of respiratory virus infection in humans. J Med Virol 92:408-417. https://doi.org/10.1002/jmv.25674

6. Chen Z, Li Y, Wu B et al (2020) A patient with COVID-19 presenting a false-negative reverse transcriptase polymerase chain reaction result. Korean J Radiol 21:623-624. https://doi. org/10.3348/kjr.2020.0195

7. Li D, Wang D, Dong J et al (2020) False-negative results of realtime reverse-transcriptase polymerase chain reaction for severe acute respiratory syndrome coronavirus 2: role of deep-learningbased CT diagnosis and insights from two cases. Korean J Radiol 21:505-508. https://doi.org/10.3348/kjr.2020.0146

8. Diagnosis and Treatment Guideline for COVID-19 (5th edition). (2020) National Health Commission of People's Republic of China. https://www.nhc.gov.cn/yzygj/s7653p/202002/d4b895337e 19445f8d728fcafle3e13a.shtml. Accessed May 142020

9. Wang D, Hu B, Hu C et al (2020) Clinical characteristics of 138 hospitalized patients with 2019 novel coronavirus-infected pneumonia in Wuhan, China. JAMA 323:1061-1069. https://doi. org/10.1001/jama.2020.1585

10. Ruan Q, Yang K, Wang W et al (2020) Clinical predictors of mortality due to COVID-19 based on an analysis of data of 150 patients from Wuhan, China. Intens Care Med 46:846-848. https ://doi.org/10.1007/s00134-020-05991-x

11. Sun H, Ning R, Tao Y et al (2020) Risk factors for mortality in 244 older adults with COVID-19 in Wuhan, China: a retrospective study. J Am Geriatr Soc 68:E19-E23E. https://doi.org/10.1111/ jgs. 16533

12. Wu F, Zhao S, Yu B et al (2020) A new coronavirus associated with human respiratory disease in China. Nature 579:265-269. https://doi.org/10.1038/s41586-020-2008-3

13. Wu J, Liu J, Li S et al (2020) Detection and analysis of nucleic acid in various biological samples of COVID-19 patients. Travel Med Infect Dis. https://doi.org/10.1016/j.tmaid.2020.101673

14. Liu R, Han H, Liu F et al (2020) Positive rate of RT-PCR detection of SARS-CoV-2 infection in 4880 cases from one hospital in Wuhan, China, from Jan to Feb 2020. Clin Chim Acta 505:172175. https://doi.org/10.1016/j.cca.2020.03.009

15. Zhou F, Yu T, Du R et al (2020) Clinical course and risk factors for mortality of adult inpatients with COVID-19 in Wuhan, China: a retrospective cohort study. Lancet 395:1054-1062. https://doi. org/10.1016/S0140-6736(20)30566-3

16. Zheng T, Yang C, Wang H-Y et al (2020) Clinical characteristics and outcomes of COVID-19 patients with gastrointestinal symptoms admitted to Jianghan Fangcang Shelter Hospital in Wuhan, China. J Med Virol. https://doi.org/10.1002/jmv.26146

17. Han C, Duan C, Zhang S et al (2020) Digestive symptoms in COVID-19 patients with mild disease severity: clinical presentation, stool viral RNA testing, and outcomes. Am J Gastroenterol 115:916-923. https://doi.org/10.14309/ajg.0000000000000664

Publisher's Note Springer Nature remains neutral with regard to jurisdictional claims in published maps and institutional affiliations. 